\title{
Bilateral carpal tunnel syndrome associated with interleukin 2 therapy
}

\author{
S.D. Heys, K.L.G. Mills ${ }^{1}$ and O. Eremin
}

Departments of Surgery and ${ }^{1}$ Orthopaedics, University of Aberdeen, University Medical Buildings, Foresterhill, Aberdeen $A B 92 Z B, U K$

\begin{abstract}
Summary: We report the development of synchronous bilateral carpal tunnel syndrome in a woman with metastatic colorectal cancer, undergoing treatment with recombinant interleukin 2 . A carpal tunnel decompression was carried out on the hand which was more severly affected, with a gradual recovery in median nerve function. To the best of our knowledge, this is the first reported case of carpal tunnel syndrome in association with recombinant interleukin 2.
\end{abstract}

\section{Introduction}

New strategies employed in the immunotherapy of human cancer have centred on the use of immune biological modifiers, with recombinant interleukin 2 (rIL2) attracting particular interest. Anti-tumour effects of rIL2 have been demonstrated in man, most notably against renal cell carcinoma and melanoma, ${ }^{1}$ but there has been a significant morbidity associated with this therapy including pyrexia, malaise, nausea and vomiting, skin rashes, fluid retention and neuropsychiatric disturbances such as confusion, agitation and hallucinations, reported. ${ }^{1}$ However, peripheral nerve dysfunction has not been previously documented. We have recently encountered a case of synchronous bilateral carpal tunnel syndrome occurring during treatment with rIL2.

\section{Case report}

A 69 year old woman underwent a sigmoid resection for a carcinoma of the sigmoid colon, but was noted to have multiple hepatic secondaries at that time. Two months after surgery she underwent treatment with $\mathrm{rIL} 2\left(18 \times 10^{6} \mathrm{IU} / \mathrm{m}^{2}\right.$ body surface area/24 hours), given by constant intravenous infusion for 120 hours, combined with three pulses of 5 -fluorouracil $\left(600 \mathrm{mg} / \mathrm{m}^{2}\right.$ body surface area), and folinic acid $\left(25 \mathrm{mg} / \mathrm{m}^{2}\right.$ body surface area) also given intravenously and repeated at monthly intervals. The first two pulses of rIL2 were tolerated well and a third course was commenced. However, after

Correspondence: S.D. Heys, F.R.C.S.

Accepted: 16 December 1991
96 hours of rIL2 therapy she experienced pain and paraesthesia in the index, middle and ring fingers of both hands, although more severe in the left hand (she had not previously experienced any similar symptoms). Clinical examination revealed bilateral diminished sensation to touch and pin-prick in the index, middle and radial border of the ring fingers, but worse on the left side. However, the skin of the palmar surface of the hands supplied by the palmar cutaneous branch of the median nerve (given off from the median nerve before it enters the carpal tunnel) remained intact. Motor testing revealed reduced power in the abductor pollicis brevis muscles.

At the same time, this patient also had clinical and biochemical evidence of the fluid retention that is normally associated with rIL2 therapy. Regular diuretic therapy was given throughout the rIL2 infusion, but this did not completely control the accumulation of extravascular fluid which was secondary to the 'vascular-leak' syndrome.

Following cessation of rIL2, there was no change in the clinical symptoms of bilateral median nerve dysfunction at the level of the carpal tunnel, but the symptoms and signs on the left side were more severe. It was, therefore, decided to carry out an urgent carpal tunnel decompression on the left side. Following this decompression there was a gradual improvement in symptoms and she underwent a further two cycles of rIL2 treatment, with no further problems and with a continuing clinical improvement in the carpal tunnel syndrome. Four months following the carpal tunnel decompression there were no clinical symptoms and no residual sensory or motor dysfunction, as assessed by clinical examination. 


\section{Discussion}

The morbidity and mortality of treatment with rIL2 has been previously well documented. The side effects of therapy are wide-ranging and, although dependent on the mode of administration and the total dose given, have been reported to affect almost all organ systems in the body. ${ }^{1-2}$ Neuropsychiatric effects have also been documented in patients undergoing therapy, with confusion, disorientation and hallucinations being most common. ${ }^{4}$ However, peripheral nerve dysfunction has not been reported previously.

The aetiology of these toxicities has not yet been fully defined but the increase in vascular permeability, induced by rIL2, and the associated 'vascular-leak' syndrome with a resultant leakage of sodium, water and protein, are believed to play an important role. ${ }^{5}$ This results in a loss of intravascular volume which in turn causes hypotension, decreased renal perfusion and the accumulation of protein-rich interstitial fluid in the tissues, which we, and others, have found difficult to control with diuretic therapy. ${ }^{3}$ The patient reported here also experienced a drop in systolic blood

\section{References}

1. Rosenberg, S.A., Lotze, M.T., Yang, J.C. et al. Experience with the use of high-dose interleukin-2 in the treatment of 652 cancer patients. Ann Surg 1989, 210: 474-485.

2. Webb, D.E., Austin, H.A. III, Belldegrun, A., Vaughan, E., Linehan, W.M. \& Rosenberg, S.A. Metabolic and renal effects of interleukin-2 immunotherapy for metastatic cancer. Clin Nephrol 1988, 30: 141-145.

3. West, W.H., Tauer, K.W., Yanelli, J.R., et al. Constant infusion recombinant interleukin-2 in adoptive immunotherapy of advanced cancer. $N$ Engl J Med 1987, 316: 898-905.

4. Denicoff, K.D., Rubinow, D.R., Papa, M.Z. et al. The neuropsychiatric effects of treatment with interleukin-2 and lymphokine-activated killer cells. Ann Intern Med 1987, 107: 293-300. pressure by approximately $20 \%$, and developed peripheral oedema of both hands and legs. Tissue $\frac{2}{3}$ oedema, of soft tissues and also of flexor tendons, $\underset{\varrho}{\stackrel{Q}{Q}}$ has previously been reported to play a role in $\bar{C}$ the pathogenesis of carpal tunnel syndrome. ${ }^{6-8} \stackrel{.}{\rightleftarrows}$ Although there was no macroscopic abnormality noted by the surgeon at the time of surgicalo decompression of the carpal tunnel, it is possible $\frac{\overline{\underline{\sigma}}}{\overline{\underline{N}}}$ that the generalized increase in interstitial fluid, $\frac{\bar{S}}{\overrightarrow{0}}$ associated with rIL2 treatment, had thus resulted $\stackrel{\otimes}{\varrho}$ in compression and ischaemic damage of the median nerve as it passed through the relatively $\vec{\circ}$ non-distendable oseofibrous carpal tunnel. For-. tunately, the surgical decompression carried out on $\vec{\omega}$ the side more severely affected resulted in recovery 4 months later.

With the increasing use of rIL2 in clinical practice, complications such as the one described $\infty$ here, and possibly other nerve entrapment synd- ${ }^{\circ}$

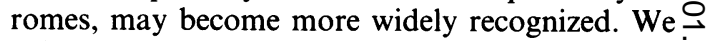
believe, therefore, that all clinicians using rIL2 therapy should be alerted to the possibility of neurological damage occurring, as well as those $\frac{}{5}$ practitioners involved in the assessment and treat $-\overrightarrow{\vec{c}}$ ment of peripheral nerve lesions.

5. Rosenstein, M., Ettinghausen, S.E. \& Rosenberg, S.A. Ext vasation of intravascular fluid mediated by the systemic administration of IL-2. J Immunol 1986, 137: 1735-1742.

6. Phalen, G. Reflections on 21 years experience with the carpal-tunnel syndrome. JAMA 1970, 212: 1365-1367.

7. Ellis, J., Folkers, K., Watanabe, T. et al. Clinical results of a $\underset{\overrightarrow{2}}{\Rightarrow}$ cross-over treatment with pyridoxine and placebo of the carpal tunnel syndrome. Am J Clin Nutr 1979, 32: 2040-2046.

8. Emara, M.K. \& Saadah, A.M. The carpal tunnel syndrome in hypertensive patients with beta-blockers. Postgrad Med $\sqrt{0}$ 1988, 64: 191-192. 